\title{
On Properties and Applications of a Two-Parameter Xgamma Distribution
}

\author{
Subhradev Sen ${ }^{1, \star}$, N. Chandra ${ }^{2}$, and Sudhansu S. Maiti ${ }^{3}$ \\ ${ }^{1}$ Alliance School of Business, Alliance University, Bengaluru, Karnataka, India \\ ${ }^{2}$ Department of Statistics, Pondicherry University, Kalapet, Puducherry, India \\ ${ }^{3}$ Department of Statistics, Visva-Bharati University, West Bengal, India
}

\section{ARTICLE INFO}

Article History

Received January 10, 2017

Accepted February 11,2018

Keywords

Lifetime distribution

maximum likelihood estimation

survival properties

reliability

2000 Mathematics Subject

Classification

$60 \mathrm{~K} 10,62 \mathrm{E} 10$

\begin{abstract}
An existing one-parameter probability distribution can be very well generalized by adding an extra parameter in it and, in turn, the two-parameter family of distributions, thus obtained, provides added flexibility in modeling real life data. In this article, we propose and study a two-parameter generalization of xgamma distribution [1] and utilize it in modeling time-to-event data sets. Along with the different structural and distributional properties of the proposed two-parameter xgamma distribution, we concentrate in studying useful survival and reliability properties, such as hazard rate, reversed hazard rate, stress-strength reliability etc. Two methods of estimation, viz. maximum likelihood and method of moments, are been suggested for estimating unknown parameters. Distributions of order statistics, stochastic order relationships are investigated for the proposed model. A Monte-Carlo simulation study is carried out to observe the trends in estimation process. Two real life time-to-event data sets are analyzed and the proposed model is compared with some other two-parameter lifetime models in the literature.
\end{abstract}

(C) 2018 The Authors. Published by Atlantis Press SARL. This is an open access article under the CC BY-NC license (http://creativecommons.org/licenses/by-nc/4.0/).

\section{INTRODUCTION}

Adding extra parameters to an existing family of distributions is very common in the statistical distribution theory as the resulting family of distributions, thus obtained, becomes richer and sometimes more flexible in modeling real life data sets. However, adding more parameters to an existing family of distributions may create complications in its basic structural properties and/or in methods of estimating the additional parameters, see for more details Johnson et al. [2]. Nevertheless, adding an extra parameter to an existing probability distribution generalizes the baseline distribution and provides flexibility in modeling or describing real life data.

Recently, Sen et al. [1] introduced and studied a one-parameter lifetime distribution, named as xgamma distribution, with probability density function (PDF) as

$$
f(x)=\frac{\theta^{2}}{(1+\theta)}\left(1+\frac{\theta}{2} x^{2}\right) e^{-\theta x}, x>0, \theta>0 .
$$

The xgamma distribution has several interesting structural and survival properties that made it useful in modeling time-to-event data sets. In another recent research paper, Sen and Chandra [3] introduced and studied different properties of a two-parameter extension or generalization of xgamma density, named as quasi xgamma distribution (QXD), and applied it in modeling bladder cancer survival data. QXD resembles closely with xgamma distribution in its density form and in other survival properties.

Our objective in this article is to introduce and study an another two-parameter generalization of xgamma distribution by adding an additional parameter $\alpha(>0)$ to it. The beauty of this two-parameter extension is that it contains xgamma distribution as a special case. We have studied different distributional, survival and/or reliability properties of this two-parameter xgamma distribution (TPXG) and demonstrated its applicability in modeling lifetime data sets with potential flexibility over existing two-parameter lifetime models. The rest of the article is organized as follows:

The TPXG along with its alternative form is introduced in section 2. The moments and related measures are studied in section 3. Incomplete moments are utilized in studying famous inequality curves and different entropy measures are studied in sections 4 and 5 , respectively. 
In section 6, different survival properties are studied. Stress-strength reliability and distributions of order statistics are described in sections 7 and 8, respectively. Section 9 studies some stochastic ordering. Methods of estimating parameters are discussed in section 10. A sample generation algorithm along with a Monte-Carlo simulation study is presented in section 11. In section 12, two real data sets are analyzed to show the applicability of TPXG. Finally, section 13 concludes.

\section{THE TPXG}

In this section we introduce and study a two-parameter form of the xgamma distribution. We have the following definition.

Definition 2.1. A continuous random variable, $X$, will be said to follow a TPXG with parameters $\alpha$ and $\theta$ if its PDF is of the form

$$
f(x)=\frac{\theta^{2}}{(\alpha+\theta)}\left(1+\frac{\alpha \theta}{2} x^{2}\right) e^{-\theta x}, x>0, \theta>0, \alpha>0 .
$$

We denote it by $X \sim \operatorname{TPXG}(\alpha, \theta)$.

Note.

1. When we put $\alpha=1$ in (2), we obtain the xgamma distribution with parameter $\theta$ as a special case.

2. The TPXG as obtained in (2) is a special mixture of exponential $(\theta)$ and gamma $(3, \theta)$ with mixing proportions $\theta /(\alpha+\theta)$ and $\alpha /(\alpha+\theta)$, respectively.

\section{Alternative form:}

An alternative form of the TPXG can be obtained by putting $\beta=1 / \alpha$ in (2) and will have the form of the PDF as

$$
f(x)=\frac{\theta^{2}}{(1+\beta \theta)}\left(\beta+\frac{\theta}{2} x^{2}\right) e^{-\theta x}, x>0, \theta>0, \beta>0 .
$$

The cumulative distribution function (CDF) of $X$ as given in 2 is given by

$$
F(x)=1-\frac{\left(\alpha+\theta+\alpha \theta x+\frac{1}{2} \alpha \theta^{2} x^{2}\right)}{(\alpha+\theta)} e^{-\theta x}, x>0, \theta>0, \alpha>0 .
$$

The characteristic function $(\mathrm{CF})$ of $X$ is derived as

$$
\phi_{X}(t)=E\left[e^{i t X}\right]=\frac{\theta^{2}}{(\alpha+\theta)}\left[(\theta-i t)^{-1}+\alpha \theta(\theta-i t)^{-3}\right] ; t \in \mathbb{R}, i=\sqrt{-1} .
$$

The plot of probability density curves for different values of $\alpha$ and $\theta$ is shown in Figure 1 .

\section{MOMENTS AND RELATED MEASURES}

In this section we study the moments and other related measures for the TPXG with parameters $\alpha$ and $\theta$, i.e., TPXG ( $\alpha, \theta)$.

The $r^{\text {th }}$ order raw moments for $X \sim \operatorname{TPXG}(\alpha, \theta)$ is obtained as

$$
\begin{aligned}
\mu_{r}^{\prime} & =E\left(X^{r}\right)=\int_{0}^{\infty} x^{r} \frac{\theta^{2}}{(\alpha+\theta)}\left(1+\frac{\alpha \theta}{2} x^{2}\right) e^{-\theta x} d x \\
& =\frac{r !}{2 \theta^{r}(\alpha+\theta)}[2 \theta+\alpha(1+r)(2+r)] ; r=1,2, \ldots
\end{aligned}
$$

In particular, we have,

$$
\mu_{1}^{\prime}=E(X)=\frac{(\theta+3 \alpha)}{\theta(\alpha+\theta)} \quad ; \quad \mu_{2}^{\prime}=E\left(X^{2}\right)=\frac{2(\theta+6 \alpha)}{\theta^{2}(\alpha+\theta)} .
$$

So, we have the expression for second order central (about mean) moment or the population variance for $X$ as

$$
V(X)=\mu_{2}=\frac{2\left(\theta^{2}+8 \alpha \theta+3 \alpha^{2}\right)}{\theta^{2}(\alpha+\theta)^{2}}
$$



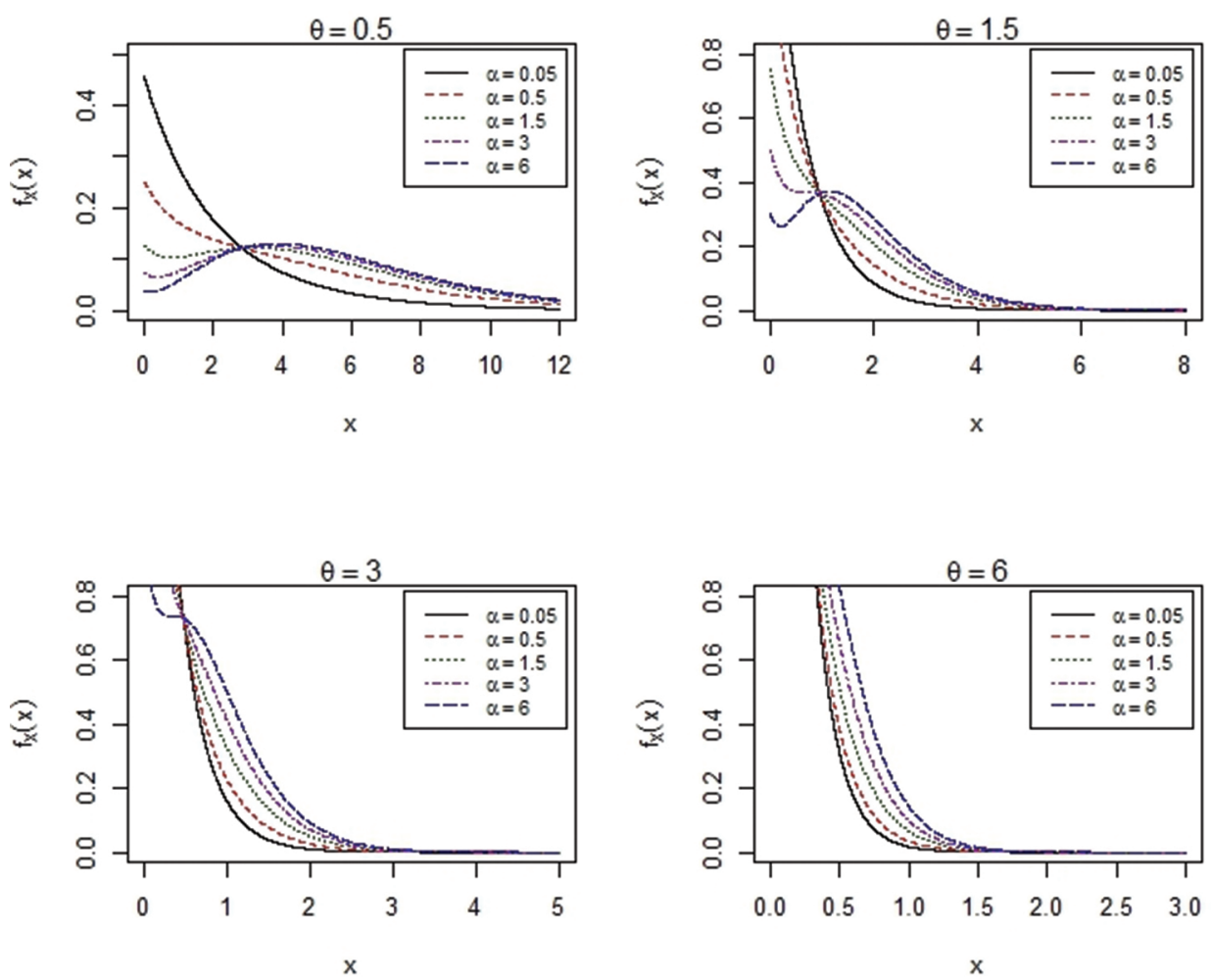

Figure 1 Probability density function of two-parameter xgamma distribution for different values of $\alpha$ and $\theta$.

so that the coefficient of variation $(\mathrm{CV})$ becomes

$$
\gamma=\frac{\sqrt{2\left(\theta^{2}+8 \alpha \theta+3 \alpha^{2}\right)}}{(\theta+3 \alpha)}
$$

The moment generating function (MGF) of $X$ is derived as

$$
M_{X}(t)=E\left[e^{t X}\right]=\frac{\theta^{2}}{(\alpha+\theta)}\left[(\theta-t)^{-1}+\alpha \theta(\theta-t)^{-3}\right] ; t \in \mathbb{R} .
$$

The cumulant generating function (CGF) of $X$ is obtained as

$$
K_{X}(t)=\ln M_{X}(t)=\ln \frac{\theta^{2}}{(\alpha+\theta)(\theta-t)}+\ln \left[1+\frac{\alpha \theta}{(\theta-t)^{2}}\right] ; t \in \mathbb{R} .
$$

The following theorem shows that $\operatorname{TPXG}(\alpha, \theta)$ is unimodal.

Theorem 3.1. For $\theta>\alpha / 2$, the PDF, $f(x)$ of $X \sim T P X G(\alpha, \theta)$, as given in (2), is decreasing in $x$.

Proof. We have from (2) the first derivative of $f(x)$ with respect to $x$ as

$$
f^{\prime}(x)=\frac{\theta^{2}}{(\alpha+\theta)}\left(\alpha \theta x-\theta-\frac{1}{2} \alpha \theta^{2} x^{2}\right) e^{-\theta x} .
$$

$f^{\prime}(x)$ is negative in $x$ when $\theta>\alpha / 2$, and hence the proof.

So, we have from the theorem 3.1, for $\theta \leq \alpha / 2, \frac{d}{d x} f(x)=0$ which implies that $\left(1,+, \sqrt{1-2 \frac{\theta}{\alpha}}\right) / \theta$ is the unique critical point at which $f(x)$ is maximized. 
Hence, the mode of TPXG $(\alpha, \theta)$ is given by

$$
\operatorname{Mode}(X)= \begin{cases}\frac{1+\sqrt{1-\frac{2 \theta}{\alpha}}}{\theta}, & \text { if } 0<\theta \leq \alpha / 2 . \\ 0, & \text { otherwise. }\end{cases}
$$

\section{INCOMPLETE MOMENTS AND INEQUALITY CURVES}

The $r^{\text {th }}$ incomplete moment, $\mu_{r}(t)$ (say), for a random variable $X$ with $\operatorname{PDF} f(x)$ is defined as

$$
\mu_{r}(t)=\int_{0}^{t} x^{r} f(x) d x
$$

When $X \sim \operatorname{TPXG}(\alpha, \theta)$, the $r^{\text {th }}$ incomplete moment is obtained as

$$
\begin{aligned}
\mu_{r}(t) & =\frac{\theta^{2}}{(\alpha+\theta)} \int_{0}^{t} x^{r}\left(1+\frac{\alpha \theta}{2} x^{2}\right) e^{-\theta x} d x \\
& =\frac{\theta^{2}}{(\alpha+\theta)}\left[\gamma(r+1, \theta t)+\frac{\alpha \theta}{2} \gamma(r+3, \theta t)\right],
\end{aligned}
$$

where $\gamma(a, x)=\int_{0}^{x} u^{a-1} e^{-u} d u$ is lower incomplete gamma function.

Lorenz curve and Bonferroni curve are well known inequality curves (see for more details Kleiber and Kotz [4]) that have been extensively applied in many fields such as economics, demography, insurance, medicine and reliability engineering.

When a non-negative continuous random variable $X$ has $\operatorname{PDF} f(x)$ and CDF $F(x)$, the Lorenz and Bonferroni curves are defined by

$$
L(p)=\frac{1}{\mu} \int_{0}^{q} x f(x) d x
$$

and

$$
B(p)=\frac{1}{p \mu} \int_{0}^{q} x f(x) d x
$$

respectively, where $\mu=E(X)$ and $q=F^{-1}(p)$ for $0<p<1$.

When $X \sim \operatorname{TPXG}(\alpha, \theta)$, we use the first incomplete moment putting $r=1$ in (14) to obtain Lorenz and Bonferroni curves as

$$
L(p)=\frac{\theta^{3}}{(3 \alpha+\theta)}\left[\gamma(2, \theta q)+\frac{\alpha \theta}{2} \gamma(4, \theta q)\right]
$$

and

$$
B(p)=\frac{\theta^{3}}{p(3 \alpha+\theta)}\left[\gamma(2, \theta q)+\frac{\alpha \theta}{2} \gamma(4, \theta q)\right],
$$

respectively, where, for given $p, \alpha$ and $\theta, q$ is the solution of the equation, $\gamma(1, \theta q)+\frac{\alpha \theta}{2} \gamma(3, \theta q)=\frac{p(\alpha+\theta)}{\theta^{2}}$ that can easily be solved numerically.

\section{ENTROPY MEASURES}

An entropy of a random variable $X$ is a measure of variation of the uncertainty. A popular entropy measure is Rényi entropy. If a non-negative continuous random variable, $X$, has the $\operatorname{PDF} f(x)$, then Rényi entropy is defined as

$$
H_{R}(\gamma)=\frac{1}{1-\gamma} \ln \int_{0}^{\infty} f^{\gamma}(x) d x \text { for } \gamma>0(\neq 1) .
$$


When $X \sim \operatorname{TPXG}(\alpha, \theta)$, one can derive

$$
\int_{0}^{\infty} f^{\gamma}(x) d x=\frac{\theta^{2 \gamma}}{(\alpha+\theta)^{\gamma}} \sum_{j=0}^{\gamma}\left(\begin{array}{l}
\gamma \\
j
\end{array}\right)\left(\frac{\alpha}{2}\right)^{j} \frac{\Gamma(2 j+1)}{\theta^{j+1} \gamma^{2 j+1}}
$$

to obtain Rényi entropy as

$$
H_{R}(\gamma)=\frac{1}{1-\gamma}[2 \gamma \ln \theta-\gamma \ln (\alpha+\theta)]+\frac{1}{1-\gamma} \ln \left[\sum_{j=0}^{\gamma}\left(\begin{array}{l}
\gamma \\
j
\end{array}\right)\left(\frac{\alpha}{2}\right)^{j} \frac{\Gamma(2 j+1)}{\theta^{j+1} \gamma^{2 j+1}}\right]
$$

In physics, the Tsallis entropy[5] is a generalization of the standard Boltzmann-Gibbs entropy. For an absolutely continuous non-negative random variable $X$ with $\operatorname{PDF} f(x)$, Tsallis entropy (also called q-entropy) is definedas

$$
S_{q}(X)=\frac{1}{q-1} \ln \left[1-\int_{0}^{\infty} f^{q}(x) d x\right] \text { for } q>0(\neq 1)
$$

When $X \sim \operatorname{TPXG}(\alpha, \theta)$, Tsallis entropy can be derived as

$$
S_{q}(X)=\frac{1}{q-1} \ln \left[1-\frac{\theta^{2 q}}{(\alpha+\theta)^{q}} \sum_{j=0}^{q}\left(\begin{array}{c}
q \\
j
\end{array}\right)\left(\frac{\alpha}{2}\right)^{j} \frac{\Gamma(2 j+1)}{\theta^{j+1} q^{2 j+1}}\right] .
$$

Shannon measure of entropy is defined as

$$
H(f)=E[-\ln f(x)]=-\int_{0}^{\infty} \ln [f(x)] f(x) d x
$$

For $X \sim \operatorname{TPXG}(\alpha, \theta)$, Shannon entropy is obtained as

$$
H(f)=\left(\frac{3 \alpha+\theta}{\alpha+\theta}\right)-\ln \frac{\theta^{2}}{(\alpha+\theta)}-\frac{\theta^{2}}{(\alpha+\theta)} \sum_{j=1}^{\infty}(-1)^{j+1} \frac{(\alpha / 2)^{j}}{\theta^{j+1} j}\left[\Gamma(2 j+1)+\frac{\alpha}{2 \theta} \Gamma(2 j+3)\right] .
$$

\section{SURVIVAL PROPERTIES}

In this section we study different survival properties of the TPXG with parameters $\alpha$ and $\theta$ given in (2).

The survival function (SF) of $X$ is given by

$$
S(x)=\frac{\left(\alpha+\theta+\alpha \theta x+\frac{1}{2} \alpha \theta^{2} x^{2}\right)}{(\alpha+\theta)} e^{-\theta x} ; x>0, \theta>0, \alpha>0 .
$$

The hazard rate (HR) function of $X$ is obtained as

$$
h(x)=\frac{f(x)}{S(x)}=\frac{\theta^{2}\left(1+\frac{\alpha \theta}{2} x^{2}\right)}{\left(\alpha+\theta+\alpha \theta x+\frac{1}{2} \alpha \theta^{2} x^{2}\right)} ; x>0, \theta>0, \alpha>0 .
$$

Note. The HR function, $h(x)$, is increasing for $x>\sqrt{\frac{2}{\alpha \theta}}$ (see Figure 2 for HR plot for different values of $\alpha$ and $\theta$ ) with the following bounds.

$$
f(0)=\frac{\theta^{2}}{(\alpha+\theta)}<h(x)<\theta
$$

Theorem 6.1. The failure rate, $h(x)$, as given in (21) is increasing failure rate (IFR) in distribution for $x>\sqrt{\frac{2}{\alpha \theta}}$ and is decreasing failure rate (DFR) in distribution for $x<\sqrt{\frac{2}{\alpha \theta}}$.

Proof. The proof comes immediately as the PDF given in (2) is log-concave for $x>\sqrt{\frac{2}{\alpha \theta}}$ and log-convex for $x<\sqrt{\frac{2}{\alpha \theta}}$. 

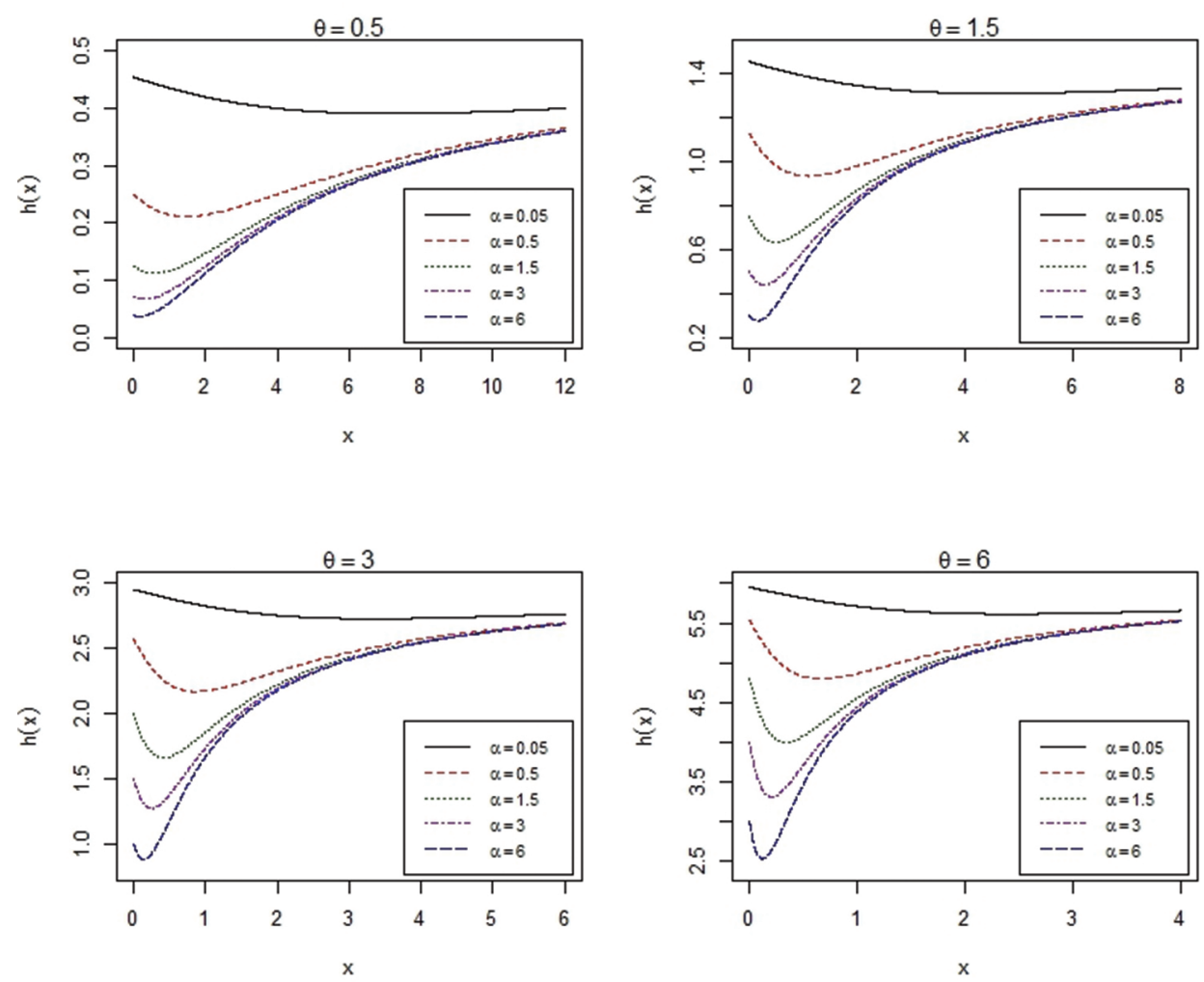

Figure 2 Hazard rate function of two-parameter xgamma distribution for different values of $\alpha$ and $\theta$.

The reversed hazard rate (RHR) function of $X$ is obtained as

$$
r(x)=\frac{f(x)}{F(x)}=\frac{\theta^{2}\left(1+\frac{\alpha \theta}{2} x^{2}\right) e^{-\theta x}}{(\alpha+\theta)\left(1-e^{-\theta x}\right)-\left(1+\frac{\theta x}{2}\right) \alpha \theta x e^{-\theta x}} ; x>0, \theta>0, \alpha>0 .
$$

The mean residual life (MRL) function of $X$ is given by

$$
\begin{aligned}
m(x) & =E[X-x \mid X>x]=\frac{1}{S(x)} \int_{x}^{\infty} S(t) d t \\
& =\frac{1}{\theta}+\frac{\alpha(2+\theta x)}{\theta\left(\alpha+\theta+\alpha \theta x+\frac{1}{2} \alpha \theta^{2} x^{2}\right)} .
\end{aligned}
$$

Note. The MRL function, $m(x)$, is bounded with the following limits,

$$
\frac{1}{\theta}<m(x)<\frac{(\theta+3 \alpha)}{\theta(\alpha+\theta)}=E(X) .
$$

\section{STRESS-STRENGTH RELIABILITY}

Let $X$ and $Y$ be continuous random variables denote strength and stress, respectively, of an equipment or a system, then the stress-strength reliability is defined as

$$
R=\operatorname{Pr}(X>Y)=\int_{0}^{\infty} \operatorname{Pr}(X>Y \mid Y=y) f_{Y}(y) d y=\int_{0}^{\infty} S_{X}(y) f_{Y}(y) d y,
$$

where $f_{Y}($.$) is the PDF of Y$ and $S_{X}($.$) is the SF of X$. 
If $X \sim \operatorname{TPXG}\left(\alpha_{1}, \theta_{1}\right)$ and $Y \sim \operatorname{TPXG}\left(\alpha_{2}, \theta_{2}\right)$ independently, then stress-strength reliability is obtained as

$$
\begin{gathered}
R=\frac{\theta_{2}^{2}}{\left(\alpha_{1}+\theta_{1}\right)\left(\alpha_{2}+\theta_{2}\right)}\left[\left(\frac{\alpha_{1}+\theta_{1}}{\theta_{1}+\theta_{2}}\right)+\frac{\alpha_{1} \theta_{1}}{\left(\theta_{1}+\theta_{2}\right)^{2}}+\frac{\alpha_{1} \alpha_{2} \theta_{2}+\alpha_{2} \theta_{1} \theta_{2}+\alpha_{1} \theta_{1}^{2}}{\left(\theta_{1}+\theta_{2}\right)^{3}}\right] \\
+\frac{3 \alpha_{1} \alpha_{2} \theta_{1} \theta_{2}^{3}}{\left(\alpha_{1}+\theta_{1}\right)\left(\alpha_{2}+\theta_{2}\right)\left(\theta_{1}+\theta_{2}\right)^{4}}+\frac{6 \alpha_{1} \alpha_{2} \theta_{1}^{2} \theta_{2}^{3}}{\left(\alpha_{1}+\theta_{1}\right)\left(\alpha_{2}+\theta_{2}\right)\left(\theta_{1}+\theta_{2}\right)^{5}}
\end{gathered}
$$

In particular, when $X$ and $Y$ are independently and identically distributed (IID) TPXG $(\alpha, \theta)$, we have the expression for stress-strength reliability as

$$
R=\frac{\theta^{2}}{(\alpha+\theta)^{2}}\left[\frac{\alpha}{\theta}+\frac{1}{2}+\frac{\alpha^{2}}{2 \theta^{2}}\right]
$$

Note. If we put $\alpha=1$ in (25), we have $R=1 / 2$, which is nothing but the stress-strength reliability when $X$ and $Y$ are IID $x g a m m a(\theta)$.

\section{DISTRIBUTION OF ORDER STATISTICS}

Distributions of order statistics for a lifetime random variable play important roles in computing system reliability in case of series or parallel configurations with IID components.

Let $X_{1}, X_{2}, \ldots, X_{n}$ be a random sample of size $n$ drawn from $X \sim \operatorname{TPXG}(\alpha, \theta)$.

Denote $X_{(j)}$ as the $j^{\text {th }}$ order statistic. Then $X_{(1)}$ and $X_{(n)}$ denote the smallest and largest order statistics for a sample of size $n$ drawn from $\operatorname{TPXG}(\alpha, \theta)$, respectively.

The PDF of $X_{(1)}$ is derived as

$$
\begin{aligned}
f_{X_{(1)}}(x) & =n[1-F(x)]^{n-1} f(x) \\
& =\frac{n \theta^{2}}{(\alpha+\theta)^{n}}\left(1+\frac{\alpha \theta}{2} x^{2}\right)\left[\alpha+\theta+\alpha \theta x+\frac{1}{2} \alpha \theta^{2} x^{2}\right]^{n-1} e^{-n \theta x}
\end{aligned}
$$

for $x>0, \theta>0$ and $\alpha>0$.

Similarly, the PDF of $X_{(n)}$ is obtained as

$$
\begin{aligned}
f_{X_{(n)}}(x) & =n[F(x)]^{n-1} f(x) \\
& =\frac{n \theta^{2}}{(\alpha+\theta)^{n}}\left(1+\frac{\alpha \theta}{2} x^{2}\right)\left[(\alpha+\theta)\left(1-e^{-\theta x}\right)-\left(1+\frac{\theta x}{2}\right) \alpha \theta x e^{-\theta x}\right]^{n-1} e^{-\theta x}
\end{aligned}
$$

for $x>0, \theta>0$ and $\alpha>0$.

\section{STOCHASTIC ORDERING}

In this section we study stochastic ordering relations for random variables following TPXG $(\alpha, \theta)$. Stochastic ordering is an important tool for judging the comparative behavior. Recall some basic definitions.

Definition 9.1. A non-negative random variable $\mathrm{X}_{1}$ is said to be smaller than an another non-negative random variable $X_{2}$ in the
i. $\quad$ stochastic order $\left(X_{1} \leq_{S T} X_{2}\right)$ if $F_{X_{1}}(x) \geq F_{X_{2}}(x)$ for all $x$.
ii. HR order $\left(X_{1} \leq_{H R} X_{2}\right)$ if $h_{X_{1}}(x) \geq h_{X_{2}}(x)$ for all $x$.
iii. $\quad$ MRL order $\left(X_{1} \leq_{M R L} X_{2}\right)$ if $m_{X_{1}}(x) \leq m_{X_{2}}(x)$ for all $x$.
iv. likelihood ratio order $\left(X_{1} \leq_{L R} X_{2}\right)$ if $\frac{f_{X_{1}}(x)}{f_{X_{2}}(x)}$ decreases in $x$. 
The following implications [6] are well justified:

$$
\begin{gathered}
X_{1} \leq_{L R} X_{2} \Rightarrow X_{1} \leq_{H R} X_{2} \Rightarrow X \leq_{M R L} X_{2} \text { and } \\
X \leq_{H R} X_{2} \Rightarrow X \leq_{S T} X_{2} .
\end{gathered}
$$

The following theorems shows that the TPXG is ordered with respect to the strongest likelihood ratio ordering and thereby the other orderings as mentioned in definition 9.1 .

Theorem 9.1. Let $X_{1} \sim \operatorname{TPXG}\left(\alpha_{1}, \theta_{1}\right)$ and $X_{2} \sim \operatorname{TPXG}\left(\alpha_{2}, \theta_{2}\right)$. If $\alpha_{1}=\alpha_{2}$ and $\theta_{1} \geq \theta_{2}$ (or, if $\theta_{1}=\theta_{2}$ and $\left.\alpha_{1} \leq \alpha_{2}\right)$, then $X_{1} \leq{ }_{L R} X_{2}$ and hence $X_{1} \leq_{H R} X_{2}, X_{1} \leq_{M R L} X_{2}$ and $X_{1} \leq_{S T} X_{2}$.

Proof. Let us denote the PDF of $X_{1}$ as $f_{X_{1}}(x)$ and that of $X_{2}$ be $f_{X_{2}}(x)$ for $x>0$.

We have then the ratio

$$
\frac{f_{X_{1}}(x)}{f_{X_{2}}(x)}=\frac{\theta_{1}^{2}\left(\alpha_{2}+\theta_{2}\right)}{\theta_{2}^{2}\left(\alpha_{1}+\theta_{1}\right)}\left(\frac{1+\frac{\alpha_{1} \theta_{1}}{2} x^{2}}{1+\frac{\alpha_{2} \theta_{2}}{2} x^{2}}\right) e^{-\left(\theta_{1}-\theta_{2}\right) x}
$$

Taking logarithm both sides, we have

$$
\ln \left[\frac{f_{X_{1}}(x)}{f_{X_{2}}(x)}\right]=2 \ln \left(\frac{\theta_{1}}{\theta_{2}}\right)+\ln \left(\frac{\alpha_{2}+\theta_{2}}{\alpha_{1}+\theta_{1}}\right)+\ln \left(\frac{1+\frac{\alpha_{1} \theta_{1}}{2} x^{2}}{1+\frac{\alpha_{2} \theta_{2}}{2} x^{2}}\right)-\left(\theta_{1}-\theta_{2}\right) x .
$$

The first derivative with respect to $x$ gives

$$
\frac{d}{d x} \ln \left[\frac{f_{X_{1}}(x)}{f_{X_{2}}(x)}\right]=\frac{\left(\alpha_{1} \theta_{1}-\alpha_{2} \theta_{2}\right) x}{\left(1+\frac{\alpha_{1} \theta_{1}}{2} x^{2}\right)\left(1+\frac{\alpha_{2} \theta_{2}}{2} x^{2}\right)}-\left(\theta_{1}-\theta_{2}\right),
$$

which is negative when $\alpha_{1}=\alpha_{2}$ and $\theta_{1} \geq \theta_{2}$ (or, when $\theta_{1}=\theta_{2}$ and $\alpha_{1} \leq \alpha_{2}$ ), i.e., $\frac{f_{X_{1}}(x)}{f_{X_{2}}(x)}$ decreases in $x$ when $\alpha_{1}=\alpha_{2}$ and $\theta_{1} \geq \theta_{2}$ (or, when $\theta_{1}=\theta_{2}$ and $\left.\alpha_{1} \leq \alpha_{2}\right)$, so $X_{1} \leq_{L R} X_{2}$ and the other orderings follow automatically by (28). Hence the proof.

Now, we establish stochastic order relationships between two random variables, $X$ and $Y$, when $X \sim T P X G\left(\alpha_{1}, \theta_{1}\right)$ and $Y \sim Q X D\left(\alpha_{2}, \theta_{2}\right)$. Note that the QXD with parameters $\alpha$ and $\theta$ has the PDF

$$
f(x)=\frac{\theta}{(1+\alpha)}\left(\alpha+\frac{\theta^{2}}{2} x^{2}\right) e^{-\theta x} ; x>0, \alpha, \theta>0
$$

We have the following theorem.

Theorem 9.2. Let $X \sim \operatorname{TPXG}\left(\alpha_{1}, \theta_{1}\right)$ and $Y \sim \operatorname{QXD}\left(\alpha_{2}, \theta_{2}\right)$. If $\alpha_{1}=\alpha_{2}=\alpha\left(\right.$ say), then $X \leq_{L R} Y$ whenever $\frac{\left(\theta_{1}-\theta_{2}\right)+\theta_{2}^{2}}{\theta_{1}} \geq \alpha^{2}$ and $\theta_{1}>\theta_{2}$. Again, if $\theta_{1}=\theta_{2}=\theta$ (say), then $X \leq_{L R} Y$ whenever $\alpha_{1} \leq \frac{\theta}{\alpha_{2}}$.

Proof. The proof comes immediately following the similar arguments as followed in the proof of theorem 9.1. Hence is omitted.

\section{ESTIMATION OF PARAMETERS}

In this section we propose method of moments and maximum likelihood estimators (MLEs) for and $\theta$ when $X \sim T P X G(\alpha, \theta)$.

Let $X_{1}, X_{2}, \ldots, X_{n}$ be a random sample of size $n$ drawn from TPXG $(\alpha, \theta)$. Denote $\bar{X}$ as sample mean.

\subsection{Method of Moments Estimation}

Using the first two raw moments given in (7), we have

$$
\frac{\mu_{2}^{\prime 2}}{\mu_{1}^{\prime}}=\frac{2(\theta+6 \alpha)(\alpha+\theta)}{(\theta+3 \alpha)^{2}}=k(\text { say })
$$


Taking $=\mathrm{c} \alpha$, we have

$$
\frac{\mu_{2}^{\prime 2}}{\mu_{1}^{\prime}}=\frac{2(c+6)(c+1)}{(c+3)^{2}}=k
$$

which give a quadratic equation in $c$ as

$$
(2-k) c^{2}+(14-6 k) c+(12-9 k)=0
$$

An estimate of $k$ is easily obtained by replacing $\mu_{1}{ }^{\prime}$ and $\mu_{2}{ }^{\prime}$ by sample moments $\bar{X}$ and $m_{2}{ }^{\prime}$, respectively, in equation (29). This estimate can then be utilized to solve (30) to obtain an estimate of $c$. Again, from the first moment equation, we have

$$
\bar{X}=\frac{(c+3)}{\alpha c(c+1)}
$$

and thus moment estimator of $\alpha, \tilde{\alpha}$ (say), is given by

$$
\tilde{\alpha}=\left[\frac{(c+3)}{c(c+1)}\right] \frac{1}{\bar{X}}
$$

Finally, the moment estimator, $\tilde{\theta}$ (say), of $\theta$ is obtained as

$$
\tilde{\theta}=\left(\frac{c+3}{c+1}\right) \frac{1}{\bar{X}}
$$

\subsection{Maximum Likelihood Estimation}

Let $x=\left(x_{1}, x_{2}, \ldots, x_{n}\right)$ be $n$ observations or realizations on a random sample $X_{1}, X_{2}, \ldots, X_{n}$ of size $n$ drawn from $X \sim T P X G(\alpha, \theta)$. We have the likelihood function as

$$
L(\alpha, \theta \mid \mathrm{X})=\prod_{i=1}^{n} \frac{\theta^{2}}{(\alpha+\theta)}\left(1+\frac{\alpha \theta}{2} x_{i}^{2}\right) e^{-\theta x_{i}}=\frac{\theta^{2 n}}{(\alpha+\theta)^{n}} e^{-\theta^{\Sigma_{i=1}^{n} x_{i}}} \prod_{i=1}^{n}\left(1+\frac{\alpha \theta}{2} x_{i}^{2}\right)
$$

The log-likelihood function is given by

$$
\ln L(\alpha, \theta \mid \mathrm{x})=2 n \ln \theta-n \ln (\alpha+\theta)-\theta\left(\sum_{i=1}^{n} x_{i}\right)+\sum_{i=1}^{n} \operatorname{In}\left(1+\frac{\alpha \theta}{2} x_{i}^{2}\right) .
$$

To find out the MLEs, $\hat{\alpha}$ and $\hat{\theta}$, of $\alpha$ and $\theta$, we have two log-likelihood equations as

$$
\frac{\partial \ln L(\alpha, \theta \mid \mathrm{x})}{\partial \alpha}=\sum_{i=1}^{n}\left(\frac{\frac{\theta}{2} x_{i}^{2}}{1+\frac{\alpha \theta}{2} x_{i}^{2}}\right)-\frac{n}{(\alpha+\theta)}=0
$$

and

$$
\frac{\partial \ln L(\alpha, \theta \mid \mathrm{x})}{\partial \theta}=\frac{2 n}{\theta}-\frac{n}{(\alpha+\theta)}+\sum_{i=1}^{n}\left(\frac{\frac{\alpha}{2} x_{i}^{2}}{1+\frac{\alpha \theta}{2} x_{i}^{2}}\right)-\sum_{i=1}^{n} x_{i}=0,
$$

respectively.

Though the log-likelihood equations cannot be solved analytically, we can utilize numerical method for solving (34) and (35) to obtain the MLEs, $\hat{\alpha}$ and $\hat{\theta}$, respectively. 


\section{SAMPLE GENERATION AND SIMULATION STUDY}

This section deals with the random sample generation algorithm for generating random samples of specific size from the TPXG. We make use of the fact that the distribution as given in (2), is a special mixtures of exponential $(\theta)$ and gamma $(3, \theta)$ for describing sample generation algorithm. To generate a random sample of size $n$ from $\operatorname{TPXG}(\alpha, 0 a)$, we have the following simulation algorithm:

i. Generate $U_{i} \sim$ uniform $(0,1), i=1,2, \ldots, n$.

ii. Generate $V_{i} \sim \operatorname{exponential}(\theta), i=1,2, \ldots, n$.

iii. Generate $W_{i} \sim \operatorname{gamma}(3, \theta), i=1,2, \ldots, n$.

iv. If $U_{i} \leq \frac{\theta}{\alpha+\theta}$, then set $X_{i}=V_{i}$, otherwise, set $X_{i}=W_{i}$.

A Monte-Carlo simulation study was carried out considering $N=10000$ times for selected values of $n, \alpha$ and $\theta$. Samples of sizes 20, 30, 50, 80 and 100 were considered and values of $(\alpha, \theta)$ were taken as $(0.5,0.5),(1.5,2.0)$ and $(3.0,4.0)$.

The following measures were computed:

(a) Average mean square error (MSE) of the simulated estimates $\hat{\alpha}_{i}, i=1,2, \ldots, N$ :

$$
\hat{\alpha}=\frac{1}{N} \sum_{i=1}^{N}\left(\hat{\alpha}_{i}-\alpha\right)^{2}
$$

(b) Average MSE of the simulated estimates $\hat{\theta}_{i}, i=1,2, \ldots, N$ :

$$
\hat{\theta}=\frac{1}{N} \sum_{i=1}^{N}\left(\hat{\theta}_{i}-\theta\right)^{2}
$$

The results of the simulation study are shown in Table 1. The following observations are made from the simulation study:

i. The estimates of $\alpha$ and $\theta$ get closer to the corresponding true values as the sample size, $n$, increases.

ii. The average MSEs for estimates of $\alpha$ and estimates $\theta$ decrease with increasing sample size.

\section{APPLICATION WITH REAL LIFE DATA ILLUSTRATION}

In this section we analyze two different time-to-event data sets for illustrating the applicability of TPXG. For comparison purpose, besides TPXG, we consider five other two parameter lifetime distributions, viz., gamma distribution with shape $\alpha$ and rate $\theta$, weibull distribution with shape $\alpha$ and scale $\beta, \log$-normal distribution with parameters $\mu$ and $\sigma$, two-parameter Lindley distribution (TPLD) with parameters alph $a$ and $\lambda$ (Shanker et al. [7] and QXD with parameters $\alpha$ and $\theta[3]$.

In order to compare the two distribution models, we consider criteria like, -log-likelihood, AIC (Akaike information criterion, see [8]) and BIC (Bayesian information criterion, see [9]), for the data sets. The better distribution corresponds to smaller -log-likelihood, AIC and BIC values. MLE is used for estimating the model parameters for both the data sets.

Illustration I: As a first illustration we consider a data set on the failure times of an electronic device reported in Wang [10]. Table 2 represents the data of 18 failure times of an electronic device. Table 3.

shows the estimates of the model parameter(s) with standard error(s) of estimates in parenthesis and model selection criteria for the first data set.

Illustration II: As a second illustration we consider a data set on the lifetimes of a device reported in Aarset [11]. Table 4. represents the data of 50 lifetimes of a device.

Table 5 shows the estimates of the model parameter(s) with standard error(s) of estimates in parenthesis and model selection criteria for the data set represented in Table 4.

In each of the above illustration, TPXG $(\alpha, \theta)$ provides better fit (in view of -log-likelihood, AIC and BIC values) as compared to the wellknown lifetime models for the considered data set. Hence, the two-parameter extension of xgamma distribution provides flexibility in modeling real life data sets in comparison with other two-parameter lifetime distributions in the literature. 
Table 1 Estimates and average MSEs of $\alpha$ and $\theta$ for different sample sizes.

\begin{tabular}{|c|c|c|c|c|}
\hline \multicolumn{5}{|c|}{$\alpha=0.1, \theta=0.5$} \\
\hline$n$ & $\hat{\alpha}$ & MSE of $\hat{\alpha}$ & $\hat{\theta}$ & MSE of $\hat{\theta}$ \\
\hline 20 & 0.3621 & 1.3402 & 0.6597 & 0.8742 \\
\hline 50 & 0.2106 & 1.2201 & 0.5892 & 0.6420 \\
\hline 80 & 0.1976 & 1.1046 & 0.5108 & 0.5602 \\
\hline 100 & 0.1691 & 1.0042 & 0.5032 & 0.4763 \\
\hline \multicolumn{5}{|c|}{$\alpha=0.1, \theta=1.5$} \\
\hline$n$ & $\hat{\alpha}$ & MSE of $\hat{\alpha}$ & $\hat{\theta}$ & MSE of $\hat{\theta}$ \\
\hline 20 & 0.3986 & 1.8756 & 1.6942 & 0.8966 \\
\hline 50 & 0.2654 & 1.4320 & 1.5730 & 0.7021 \\
\hline 80 & 0.1976 & 1.2205 & 1.5107 & 0.4503 \\
\hline 100 & 0.1430 & 0.9986 & 1.5002 & 0.3064 \\
\hline \multicolumn{5}{|c|}{$\alpha=1.5, \theta=0.5$} \\
\hline$n$ & $\hat{\alpha}$ & MSE of $\hat{\alpha}$ & $\hat{\theta}$ & MSE of $\hat{\theta}$ \\
\hline$\overline{20}$ & 2.0166 & 2.3106 & 0.6879 & 0.9845 \\
\hline 50 & 1.9822 & 1.9658 & 0.5983 & 0.6650 \\
\hline 80 & 1.7043 & 1.4576 & 0.5127 & 0.4501 \\
\hline 100 & 1.6503 & 1.1212 & 0.5026 & 0.3326 \\
\hline \multicolumn{5}{|c|}{$\alpha=1.5, \theta=2.5$} \\
\hline$n$ & $\hat{\alpha}$ & MSE of $\hat{\alpha}$ & $\hat{\theta}$ & MSE of $\hat{\theta}$ \\
\hline 20 & 2.1551 & 3.2249 & 2.6158 & 0.5344 \\
\hline 50 & 1.9256 & 1.8867 & 2.5310 & 0.2776 \\
\hline 80 & 1.8282 & 1.4404 & 2.5100 & 0.2047 \\
\hline 100 & 1.7675 & 1.2444 & 2.5004 & 0.1753 \\
\hline \multicolumn{5}{|c|}{$\alpha=3.0, \theta=5.0$} \\
\hline$n$ & $\hat{\alpha}$ & MSE of $\hat{\alpha}$ & $\hat{\theta}$ & MSE of $\hat{\theta}$ \\
\hline 20 & 4.6542 & 2.4328 & 5.7643 & 1.2376 \\
\hline 50 & 4.1035 & 2.0122 & 5.3066 & 1.0544 \\
\hline 80 & 3.6479 & 1.8768 & 5.1006 & 0.8790 \\
\hline 100 & 3.4509 & 1.0256 & 5.0016 & 0.6504 \\
\hline
\end{tabular}

Table 2 Time to failure of 18 electronic devices.

\begin{tabular}{ccccccccccccccc}
\hline 5 & 11 & 21 & 31 & 46 & 75 & 98 & 122 & 145 & 165 & 196 & 224 & 245 & 293 & 321 \\
\hline 330 & 350 & 420 & 1103 & & &
\end{tabular}

Table 3 MLEs of model parameters and model selection criteria for failure times data of 18 electronic devices.

\begin{tabular}{|c|c|c|c|c|}
\hline Distributions & Estimate(Std. Error) & -Log-likelihood & AIC & BIC \\
\hline \multirow{2}{*}{$\operatorname{Gamma}(\alpha, \theta)$} & $\hat{\alpha}=1.1131(0.3206)$ & & & \\
\hline & $\hat{\theta}=0.0064(0.0022)$ & 2110.60 & 2225.21 & 2226.99 \\
\hline \multirow{2}{*}{ Weibull $(\alpha, \beta)$} & $\hat{\alpha}=1.1458(0.2287)$ & & & \\
\hline & $\hat{\beta}=179.69(38.6837)$ & 2110.45 & 2224.89 & 2226.67 \\
\hline \multirow{2}{*}{$\log -\operatorname{normal}(\mu, \sigma)$} & $\hat{\mu}=4.6358(0.2952)$ & & & \\
\hline & $\hat{\sigma}=1.2523(0.2087)$ & 2113.03 & 2230.07 & 2231.85 \\
\hline \multirow{2}{*}{$\operatorname{TPLD}(\alpha, \lambda)$} & $\hat{\alpha}=0.0090(0.0134)$ & & & \\
\hline & $\hat{\lambda}=0.0087(0.0024)$ & 2110.30 & 2224.59 & 2226.37 \\
\hline \multirow{2}{*}{$\operatorname{QXD}(\alpha, \theta)$} & $\hat{\alpha}=0.7251(0.5740)$ & & & \\
\hline & $\hat{\theta}=0.0125(0.0027)$ & 2110.24 & 2224.48 & 2226.26 \\
\hline \multirow{2}{*}{$\operatorname{TPXG}(\alpha, \theta)$} & $\hat{\alpha}=0.0173(0.0158)$ & & & \\
\hline & $\hat{\theta}=0.0125(0.0027)$ & 2109.62 & 2223.25 & 2225.03 \\
\hline
\end{tabular}

Table 4 Lifetimes of 50 devices.

\begin{tabular}{|c|c|c|c|c|c|c|c|c|c|c|c|c|c|c|}
\hline 0.1 & 0.2 & 1 & 1 & 1 & 1 & 1 & 2 & 3 & 6 & 7 & 11 & 12 & 18 & 18 \\
\hline 67 & 67 & 67 & 67 & 72 & 75 & 79 & 82 & 82 & 83 & 84 & 84 & 84 & 85 & 85 \\
\hline 85 & 85 & 85 & 86 & 86 & & & & & & & & & & \\
\hline
\end{tabular}

\section{CONCLUDING REMARKS}

An extra non-negative parameter is added to an existing distribution, the xgamma distribution, for studying the different properties and applications of the extended distribution, named as TPXG. There are several other standard and well established procedures in the literature for obtaining generalized two-parameter family of distributions that include baseline distribution as a special case. This article reflects one such alternative in adding extra parameter to the xgamma distribution for the purpose of generalizing the baseline density and to study 
Table 5 MLEs of model parameters and model selection criteria for data on lifetimes of 50 devices.

\begin{tabular}{cccc}
\hline Distributions & Estimate(Std. Error) & -Log-likelihood & AIC \\
\hline Gamma $(\alpha, \theta)$ & $\begin{array}{c}\hat{\alpha}=0.7990(0.1375) \\
\hat{\theta}=0.0175(0.0041)\end{array}$ & 2240.19 & 2484.38 \\
\hline Weibull $(\alpha, \beta)$ & $\begin{array}{c}\hat{\alpha}=0.9492(0.1196) \\
\hat{\beta}=44.9194(6.9458)\end{array}$ & 2486.00 & 2488.20 \\
\hline $\log \operatorname{normal}(\mu, \sigma)$ & $\begin{array}{l}\hat{\mu}=3.0790(0.2472) \\
\hat{\sigma}=1.7481(0.1748)\end{array}$ & 2241.00 & 2509.65 \\
\hline $\operatorname{TPLD}(\alpha, \lambda)$ & $\begin{array}{l}\hat{\alpha}=0.0256(0.0224) \\
\hat{\lambda}=0.0317(0.0053)\end{array}$ & 2252.82 & 2484.33 \\
\hline $\operatorname{QXX}(\alpha, \theta)$ & $\begin{array}{l}\hat{\alpha}=0.7022(0.2984) \\
\hat{\theta}=0.0476(0.0056)\end{array}$ & 2240.16 & 2478.83 \\
\hline $\operatorname{TPXG}(\alpha, \theta)$ & $\begin{array}{l}\hat{\alpha}=0.0677(0.0330) \\
\hat{\theta}=0.0476(0.0056)\end{array}$ & 2237.12 & 2488.15 \\
\hline
\end{tabular}

the general fact of added flexibility in modeling real life data sets without sacrificing much in standard estimation process. Although the article focuses in observing additional flexibility of the proposed two-parameter xgamma model over the standard two-parameter models in modeling time-to-event data sets, the proposed model might also be useful and potential in describing data sets coming from diverse areas of application owing to the fact of its lucrative structural and/or distributional properties and easy standard estimation aspects.

\section{REFERENCES}

1. S. Sen, S.S. Maiti, N. Chandra, The xgamma distribution: Statistical properties and application. J. Mod. Appl. Stat. Methods.15(1) (2016),774-788.

2. N.L. Johnson, S. Kotz, N. Balakrishnan, Continuous Univariate Distributions, vol.1, John Wiley \& Sons, New York, 1994.

3. S. Sen, N. Chandra, The quasi xgamma distribution with application in bladder cancer data. Journal of Data Science, 15(1), (2017) 61-76.

4. C. Kleiber, S. Kotz, S, Statistical Size Distributions in Economics and Actuarial Sciences, Vol. 470, John Wiley \& Sons, New York, 2003.

5. C. Tsallis, Possible generalization of Boltzmann-Gibbs statistics. J. Stat. phys.52(1-2) (1988),479-487.

6. M.Shaked, and J.G.Shanthikumar. Stochastic Orders and Their Applications. Academic Press 1994.

7. R. Shanker, S. Sharma, R. Shanker, A two-parameter Lindley distribution for modeling waiting and survival times data. Appl. Math.4(02) (2013),363-368.

8. H. Akaike, A new look at the statistical model identification. IEEE trans. Automat. Contr.19(6) (1974),716-723.

9. G. Schwarz, Estimating the dimension of a model. Ann. Stat.6(2) (1978),461-464.

10. F.K. Wang, A new model with bathtub-shaped failure rate using an additive Burr XII distribution. Reliab. Eng. Syst. Safe.70(3) (2000),305-312.

11. M.V. Aarset, How to identify a bathtub hazard rate IEEE Trans. Reliab.36(1) (1987),106-108. 